\title{
Analysis of individual differences in the size- numerosity illusion by Scalar Product Model
}

\author{
YASUHARU OKAMOTO \\ Department of Behavioral Science, Faculty of Letters, Kanazawa University, Kanazawa, Ishikawa 920
}

\begin{abstract}
Stevens and Rubin (1970) investigated the size-weight illusion using magnitude estimation method and Anderson (1970) investigated the same illusion by rating method. But, in general, both methods of magnitude estimation and rating were criticized as biased (cf. Anderson, 1981 ; Parducci, 1983). In this paper, size-numerosity illusion was investigated using simultaneous pair comparison method, which did not force the subject to make magnitude estimation or rating of the quantity, and applying the scalar product model to the data. The nonmetric solution of the analysis was very successful and indicated that the size-numerosity illusion can be described by the same information integration process as in the case of the size-weight illusion, i.e. the averaging model, and Scalar Product Model can clearly display individual differences of the illusion.
\end{abstract}

Key words: illusion, numerosity, Scalar Product Model, averaging model, information integration theory, pair comparison method, individual differences.

There are various "illusions" in psychophysical judgments. For example, Stevens and Rubin (1970) investigated the size-weight illusion using the magnitude estimation method, and showed that an object was judged heavier when its size was smaller. Anderson (1970) also investigated this illusion using the rating method. According to Anderson (1974, 1981) magnitude estimation was a biased method and lacked experimental validation. Anderson (1981) argued that the power function, which had been derived from the method of magnitude estimation, was very flexible for fitting a general class of curves and power functions with different exponents could be obtained using different experimental methods. He insisted that, contrary to these theoretical and empirical faults of the method of magnitude estimation, the rating method was validated by experimental analyses based on his information integration theory (cf. Anderson, 1974, 1981, 1982).

However, the rating method may also be biased by factors other than the physical values of the stimuli to be judged. According to Parducci's range-frequency model (cf. Parducci, 1983), rating re- flects a compromise between the range and frequency values, where the range value is determined by the relative position of the stimulus in the effective range of the stimuli, and the frequency value by the relative rank order of the presentation frequency of the stimulus.

Anyhow, the present author's main concern in this short report is not to review the criticisms on power function, rating method and other related problems in psychophysics, but to show one way to avoid the problem of linear relationship between sensation and judgment, where the data analysis is based on order relations reported by the subject. The author employed pair comparisons method, where the subject was forced to choose the subjectively more intense stimulus from a pair.

As Anderson (1970, 1981) successfully analysed the size-weight illusion data by the averaging model of the information integration theory, Eq. (1) of the model was adopted here to analyse the pair comparisons data,

$$
R=w_{1} s+w_{2} s * .
$$

In the case of the size-weight illusion 
Eq. (1) means that rating $R$ is the weighted sum of the heaviness value $s$ directly related to the physical weight and the heaviness value $s^{*}$ directly related to size. To apply Eq. (1) to the data from pair comparisons, rating $R$ is replaced by a measure $M$, which can be considered to be monotonically related to subjective intensity. In this paper, the value of $M$ for the stimulus is defined by Eq. (2).

the total number of times that

$$
\begin{gathered}
M=\text { the stimulus is chosen in the } \\
\text { pair comparisons. }
\end{gathered}
$$

This definition is valid when the pair comparison judgment is made according to the averaging model Eq. (1) (cf. appendix). Now, Eq. (1) can be rewritten as

$$
M \approx w_{1, i} s+w_{2 . t} s^{*}
$$

where $\approx$ denotes order preserving relation, and the second subscript $i$ indicates that individual differences in weight parameters are allowed. To apply Eq. (3) to the data calculated by Eq. (2), the scalar product model of Bechtel, Tucker and Chang (1971) was used with necessary modification.

Input data for the scalar product model are differences $d_{i j k}$ of subject $i$ 's choice values for stimuli $j$ and $k$. The differences may be measured by asking the subject to rate differences in preferences to stimuli. When data are obtained from choice experiment, where no ratings are required, the scalar product model can be applied to the data obtained as $M$-values defined by $\mathrm{Eq}$. (2) with individual by individual nonmetric transformations. The least squares estimates of parameters $w_{1, i}, w_{2, i}$, $s$ and $s^{*}$ which make Eq. (3) fit to the $M$-values were determined by the algorithm of Bechtel et al. (1971). That is, the matrix of $M$-values was factored into the product of matrices $A$ and $B$, where $A$ is a matrix of weight values ( $w_{1, t}$ and $\left.w_{2, i}\right)$ and $B$ a matrix of object values $(s$ and $s^{*}$ ) (cf. Keller, 1962).

In the following experiment, dots pat- terns were used as stimuli because of the following reasons;

a) The author has been interested in dots patterns as stimuli.

b) If the averaging model is one of the basic models for psychophysical illusions, then it will be possible to explain illusion in psychophysical judgment of dots patterns by this model.

c) To obtain a sufficient number of data for a pair of stimuli, dots patterns are convenient because presentation can be easily controlled by a computer system.

\section{Method}

Subjects. Ten volunteers participated individually as subjects. All were undergraduates of Kanazawa University and naive to numerosity judgment experiments.

Stimuli. The dots in a stimulus object were approximately uniformly distributed within a square frame. The sizes of frames were small or large. In a frame 30,40 , or 55 dots were distributed with no overlaps. Six combinations of the frame sizes and the numbers of dots were constructed and designated as S30, S40, S55, L30, L40, L55 where the letter S or L. means that the small or large frame was selected respectively, and the associated number represents the number of dots. For each of the six combinations of the frame size and the number of dots, one fixed distribution was used respectively, but distributions of dots patterns were independent between combinations. For example, for combination L30, the same distribution of 30 dots was used for all presentations, but the distribution for S30 was independent of the distribution for L30.

Apparatus. Two slide projectors (KODAK Ektagraphic III) were used. Stimulus pairs were presented on a translucent screen (ELMO) from the back by these projectors. Intensity of the light was reduced by a one-way mirror. The lights 
from the projectors were reflected or went through the mirror and images by both projectors were presented on the same position on the screen. The subject sat in a light room. The viewing distance was approximately $50 \mathrm{~cm}$. A pair of stimuli were arranged horizontally. The visual angle between the centers of the two stimuli in a pair was approximately $21^{\circ}$ and the small and large frames subtended about $6^{\circ} \times 6^{\circ}$ and $18^{\circ} \times 18^{\circ}$, respectively. A dot subtended about $0.24^{\circ}$. The stimulus figures were white on a blue $29^{\circ} \times 43^{\circ}$ background (SUKURA COLOR FOIL was used). In front of the subject, a small box, approximately $14 \mathrm{~cm}$ (width) $\times 10 \mathrm{~cm}$ (depth) $\times 4 \mathrm{~cm}$ (height), was laid on the table. On the top of the box, two small switches were mounted and separated horizontally about $5 \mathrm{~cm}$ from each other. The working of the apparatus and recording of the responses were controlled by a 8 -bit microcomputer system (PC8801, NEG).

Procedure. For each pair of dots patterns, the subject was instructed to judge the numerosities of dots and to choose the one which seemed to contain more dots than the other. He (or she) was asked to push down the lever of the left (or right) switch if the left (or right) pattern seemed to contain more dots than the other. On each trial, the subject was always forced to choose one of the two alternatives. The pair of stimuli were presented simultaneously to eliminate time-order error (cf. Hellstrom, 1985). To reduce biases which might be introduced by the sequence used in the pair comparison experiment, the whole presentation order of the pairs was arranged according to Ross (1934). For one set of six stimuli, Ross's method produces a sequence of length 15 , which is named sequence $\mathrm{A}$ in this paper. By reversing the stimulus positions in a pair of patterns, another sequence, sequence $B$, was generated. From these sequences, the sequence of length 90 was constructed as
Table 1

$M$ wvalues calculated by Eq. (2)

\begin{tabular}{|c|c|c|c|c|c|c|}
\hline \multirow{2}{*}{$\begin{array}{l}\text { Sub- } \\
\text { jects }\end{array}$} & \multicolumn{6}{|c|}{ Stimuli } \\
\hline & $\mathrm{S} 30$ & $S 40$ & S55 & L30 & L40 & L55 \\
\hline$A$ & $13^{\mathrm{a}}$ & $21^{b}$ & 29 & 0 & $7^{a}$ & $20^{b}$ \\
\hline$B$ & 10 & 19 & 29 & 0 & 10 & 22 \\
\hline $\mathrm{C}$ & $15^{\mathrm{n}}$ & $20^{b}$ & 29 & 0 & $9^{a}$ & $17^{\mathrm{b}}$ \\
\hline $\mathrm{D}$ & 5 & 17 & 27 & 1 & 13 & 27 \\
\hline $\mathrm{E}$ & 9 & 11 & 26 & 2 & 18 & 24 \\
\hline $\mathrm{F}$ & $12^{\mathrm{a}}$ & 19 & 30 & 0 & $7^{\mathrm{a}}$ & 22 \\
\hline $\mathrm{G}$ & $10^{\mathrm{at}}$ & 19 & 29 & 2 & $7 a$ & 23 \\
\hline $\mathrm{H}$ & $17^{\mathrm{a}}$ & $21^{b}$ & 30 & 0 & $7 a$ & $15^{a, b}$ \\
\hline I & 10 & 17 & 28 & 0 & 12 & 23 \\
\hline $\mathrm{J}$ & 6 & 16 & 29 & 1 & 13 & 25 \\
\hline
\end{tabular}

Upper indices, $a$ or $b$, to $M$-values mean that there are reversals of order relations between $M$. values and numbers of dots for the stimuli with the same indices in a row. For example, for subject A, stimulus LAO (or L55) has smaller $M$-value than stimulus $\$ 30$ (or $\$ 40$ )

ABABAB. The first 80 patterns in this sequence were presented by one projector and the other 10 patterns by the other projector. In the experiment, a subsequence of length 10 , which ranged from position 71 to position 80 in the sequence $\mathrm{ABABAB}$, was added before this main sequence of length 90 , so the total length of the sequence used in the experiment was 100. A stimulus pair was presented after brief buzzer sounding, and disappeared when the subject responded or $1 \mathrm{~s}$ elapsed after the presentation. The interval between subject's responding and the next buzzer sounding was $3 \mathrm{~s}$. An experimental session for a subject took approximately $10 \mathrm{~min}$, including instructions.

\section{Results}

The initial 10 trials were discarded as practice and responses from trial 11 to 100 were analysed. $M$-values calculated by Eq. (2) were listed in Table 1. The values of parameters in Eq. (3) which made the model fit the data of Table 1 
in the sense of least squares are displayed as Figs. 1 and 2. Points $\left(s, s^{*}\right)$ for stimuli and points $\left(w_{1, i}, w_{2, i}\right)$ for subjects were plotted in Figs. 1 and 2, respectively. The fitness of the model was evaluated by the number of pairs in which the orders of $M$-values of the data and those estimated

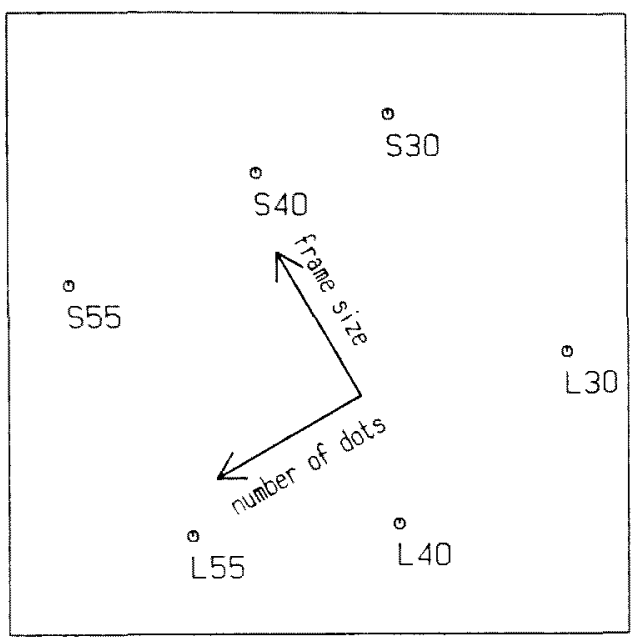

Fig. 1. The plot of the stimuli. Dimensions of the number of dots and the frame size are indicated by the arrows.

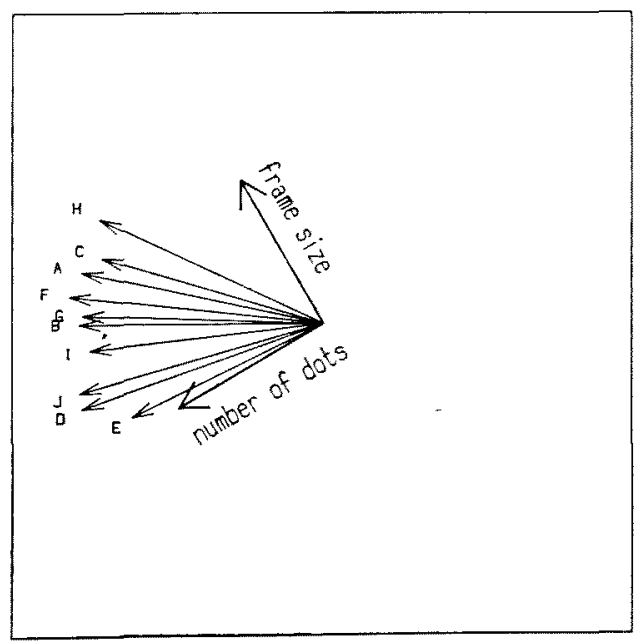

Fig. 2. The plot of the subjects. Dimensions of the number of dots and the frame size are indicated by the arrows. by the model were not compatible, because only the order information is meaningful for nonmetric transformation. The total number of pairs from 6 stimuli and 10 subjects is 150 , and for only one pair the order of $M$-values of the data did not agree with the one estimated by the model. Equation (3) fitted very well to the data.

\section{Discussion}

Table 1 shows that for each pair of stimuli of the same number of dots, the $M$-value is likely to be larger for the stimulus of the small frame than for the stimulus of the large frame. The strength of this size-numerosity illusion is not equal for all subjects. In Table 1, reversals of order relations between $M$ values and numbers of dots within a subject are indicated by upper indices, a or b. Distribution of these indices shows that the size-numerosity illusion is greater for subjects $A, C, F, G$ and $H$ than for the other subjects. Solution of Scalar Product Model displays these differences graphically. In Figs. 1 and 2, dimensions of number of dots and frame size are indicated. This interpretation of the dimensions is based on Fig. 1, where coordinates for the stimuli of the dots patterns are displayed. Scalar product used in Eq. (1) or (3) is essentially geometrical concept (cf. appendix), so solution of Scalar Product Model allows orthogonal transformation, which might make interpretation of the parameter values easier especially for more than two dimensional solution. But, in the present case, the results given in Figs. 1 and 2 can be interpreted without any transformation because the solution was given in 2-dimensional space. The configuration of stimuli in Fig. 1 shows that the numerosity as directly related to the number of dots and the numerosity as related to frame size are represented by the directions in the figures which are approxi- 
mately positively or negatively diagonal, respectively. Because of this easiness of interpretation, the solution was displayed without rotation, which would require specific criterion.

Comparison of Fig. 2 with Table 1 shows that subjects, for whom order relations of $M$-values do not agree with order relations of numbers of dots, have weight vectors $\left(w_{1, i}, w_{2, i}\right)$ with orientations closer to the frame size dimension than subjects with no disagreements. Furthermore, Fig. 2 shows the illusion qualitatively: the closer the orientation of a weight vector to the frame size dimension, the greater the size-numerosity illusion.

The preceding natural intepretations of the results of this very successful application of the model indicates that the numerosity-size illusion exists and can be described by the same averaging information integration process as in the case of the weight-size illusion.

The $M$-values of Table I were calculated by $\mathrm{Eq}$. (2) from the data of the pair comparison method, where no numerical judgments of differences in stimulus intensities were required of the subject. Without this requirement of numerical judgments, the pair comparisons method can be applied not only to adult subjects, but also to children or animals. Data from this comparison method may be analysed by Scalar Product Model as long as the model fits it. In order to apply the model successfully, individual differences in order relations of $M$-values must reflect the distribution of the stimuli in the object space. When the above conditions are met, combination of Scalar Product Model and the pair comparison method with no numerical judgments is a powerful tool.

\section{References}

Anderson, N.H. 1970 Averaging model applied to the size-weight illusion. Perception and Psychophysics, 8, 1-4.
Anderson, N.H. 1974 Algebraic models in perception. In E. G. Carterette \& M. P. Friedman (Eds.), Handbook of perception. Vol. 2. Psychophysical judgmeni and measurement. New York: Academic Press. Pp. 215-298.

Anderson, N. I. 1981 Foundations of information integration thoory. New York: Academic Press.

Anderson, N. H. 1982 Methods of information integration theory. New York: Academic Press.

Bechtel, G. G., Tucker, L. R., \& Chang, W. 1971 A scalar product model for the multidimensional scaling of choice. Psychometrika, 36, 369-388.

Hellstrom, A. 1985 The time-order error and its relatives: Mirrors of cognitive processes in comparing. Psychological Bulletin, 97, 35-61.

Keller, J. B. 1962 Factorization of matrices by least-squares. Biometrika, 49, 239-242.

Parducci, A. 1983 Category ratings and the relational character of judgment. In G. E. Stelmach \& P. A. Vroon (Eds.), Advances in psychology. Vol. 11. Modern issues in perception. Amsterdam: North-Holland. Pp. 262-282.

Ross, R.T. 1934 Optimum orders for the presentation of pairs in the method of paired comparisons. Journal of Educational Psychology, $25,375-382$.

Stevens, J. C., \& Rubin, L. L. 1970 Psychophysical scales of apparent heaviness and the size-weight illusion. Perception and Psychophysics, 8, 225-230.

(Received May 2, 1988; accepted July 15, 1989)

\section{Appendix}

$R$ value determined by Eq. (1) is scalar (or inner) product of vectors $\left(w_{1}, w_{2}\right)$ and $\left(s, s^{*}\right)$, so it is interpreted in a sense as projection of point $\left(s, s^{*}\right)$ on the line determined by vector $\left(w_{1}, w_{2}\right)$. Hence, for a fixed value of $\left(w_{1}, w_{2}\right)$, points $\left(s, s^{*}\right)$ can be assumed to be on a line when relation of $R$ values are discussed. Assume that $\mathrm{N}$ points labeled from 1 to $\mathcal{N}$ are placed in this order on a line and probability $P_{i j}$ of choosing point $i$ before point $j$ is determined by the relative position of point $i$ to point $j$, i.e.

$P_{i j} \approx$ position of point $i$-position of point $j$ where $\approx$ denotes monotonic increasing relation.

Set $M_{\alpha}$ and $M_{\beta}(\alpha<\beta)$ as follows (cf. Eq. (2));

$$
M_{\alpha}=\sum_{j \neq \alpha} P_{\alpha j} \quad M_{\beta}=\sum_{j \neq \beta} P_{\beta j}
$$

Then we have 


$$
M_{\beta}-Y_{\alpha}=\sum_{j \neq \alpha, \beta}\left(P_{\beta j}-P_{\alpha j}\right)+\left(P_{\beta, u}-P_{a \beta}\right)
$$

Since $P_{\beta j} \geq P_{\alpha j}$ and $P_{\beta a} \geq P_{a \beta}$,

$$
M_{\beta} \geq M
$$

This relation shows that $M$-value defined by Eq. (2) is monotonically related to $R$ values given by Eq. (1). 